\title{
Evaluación de la sustentabilidad de agroecosistemas familiares campesinos en la microcuenca del río Cormechoque (Boyacá)
}

\author{
Assessment of the sustainability of family farmers \\ agroecosystems in the watershed of Cormechoque \\ river (Boyacá - Colombia)
}

Fecha recepción: 22 de julio de 2015

Fecha aceptación: 3 de noviembre de 2015

Jorge Armando Fonseca-Carreño ${ }^{1}$

José Alejandro Cleves-Leguízamo²

Tomás León-Sicard ${ }^{3}$

\section{Resumen}

La investigación analizó y evaluó las prácticas agrícolas campesinas en la cuenca del río Cormechoque, en Boyacá (Colombia), y su efecto sobre el desempeño productivo, ambiental y financiero de los agroecosistemas familiares campesinos, con el objetivo de determinar el nivel de sustentabilidad de estos. A nivel metodológico se utilizó el "Marco para la Evaluación de Sistemas de Manejo de Recursos Naturales Incorporando Indicadores de Sustentabilidad" (MESMIS), a partir de la caracterización de los componentes bióticos, tecnológicos y socioeconómicos a través de seis criterios de evaluación y quince indicadores de sustentabilidad valorados en una escala desde 1 hasta 5 . Los resultados muestran que existen diferencias en la composición biológica, aspectos tecnológicos y socioeconómicos en los agroecosistemas evaluados, en razón a las diferencias en las prácticas agrícolas y la gestión de la oferta ambiental. De tal forma que el nivel de sustentabilidad de estos agroecosistemas está dado por la condición cultural de la familia rural, reflejada en su capacidad para incorporar prácticas agrícolas eficientes, mantener altos niveles de biodiversidad y una constante capacidad de aprendizaje, generando tales niveles de productividad y rentabilidad que facilita la cohesión del núcleo familiar alrededor de la actividad agropecuaria.

Palabras clave: agroecología; cuenca del río Cormechoque; indicadores de sustentabilidad; MESMIS; prácticas agrícolas.

1 M.Sc. Universidad Nacional Abierta y a Distancia (Tunja-Colombia). Estudiante Doctorado Agroecología Universidad Nacional de Colombia (Bogotá, D.C.-Colombia).jorge.fonseca@unad.edu.co.

2 Ph. D. (c) Universidad Pedagógica y Tecnológica de Colombia (Duitama, Colombia).

3 Ph. D. Universidad Nacional de Colombia (Bogotá, Colombia). teleons@unal.edu.co. 


\begin{abstract}
The research determines the sustainability level of farmers families agroecosystems in the watersheds of river Cormechoque in Boyacá (Colombia). The objective was to analyze and evaluate peasant agricultural practices and their effect on the productive, environmental and financial performance of such agroecosystems. The "Framework for assessing management systems of natural resources incorporating sustainability indicators" (MESMIS) was utilized from the biotic, technological and socio-economic components characterization, through six evaluation criteria and fifteen sustainability indicators, rated on a scale from 1 to 5 .

The results show that there are differences between the biological composition, technological and socioeconomic aspects in agroecosystems evaluated, due to differences in agricultural practices and management of environmental supply. The Tocavita agroecosystem had the highest level of sustainability due to the indicators group performance that account for the management of environmental supply (availability of water, biodiversity, soil quality), as well as cultural practices (plant cover, use of local seeds, training and dependence on external inputs) that positively impacts the profitability indicators group (Net present value and cost benefit) resulting in greater permanence of the family unit in agricultural activities.
\end{abstract}

Keywords: agricultural practices; agroecology; Cormechoque wathershed river; MESMIS; sustainability indicators. 


\section{Introducción}

La agricultura convencional en Colombia, basada en tecnologías con uso intensivo de agroinsumos y mecanización, ha generado distintos efectos sobre el ambiente y la sociedad rural, tanto en términos ecosistémicos (afectaciones a los recursos suelo, agua y biodiversidad) como socioeconómicos (concentración de recursos y migraciones) (1). Por otra parte, es evidente la compleja problemática económica y social en las zonas rurales de agricultura familiar campesina, que predominan en el centro del país; en Boyacá, esta problemática se manifiesta, entre otros aspectos, con altos niveles de pobreza, escasa escolaridad, predominio de minifundios y desplazamiento de la población rural hacia otras actividades no agropecuarias (2). Como reacción a lo anterior ha surgido un creciente interés por procurar una agricultura con mínimo impacto al ambiente y que contribuya a erradicar la pobreza (3); esta perspectiva ha incorporado el concepto de sostenibilidad (sustentabilidad, para otros autores), que desde su inclusión en el informe "Nuestro Futuro Común", de la Comisión Brundtland, en 1987, ha permeado diversos escenarios.

El concepto de sostenibilidad tiene múltiples definiciones, pero sobresalen tanto aquellas que, desde la óptica antropocentrista, la enfocan en función de satisfacer las necesidades humanas actuales sin comprometer las futuras, como las correspondientes a los enfoques donde el ecosistema puede responder a presiones drásticas, manteniendo la productividad (similar al concepto de resiliencia). La agroecología, por su parte, incorpora conceptualmente los principios de la agricultura sustentable, dando valor a las prácticas y saberes ancestrales de comunidades campesinas, afro e indígenas, orientadas a proveer la autosuficiencia alimentaria y disminuir su dependencia de insumos energéticos externos (4, 5).

Esta investigación tuvo como objetivo evaluar los niveles de sustentabilidad de agroecosistemas campesinos en la microcuenca del río Cormechoque, en el Departamento de Boyacá, usando como herramienta de análisis el "Marco para la Evaluación de Sistemas de Manejo de Recursos Naturales Incorporando Indicadores de Sustentabilidad" (MESMIS), mediante la valoración de siete criterios de desempeño estructurados a partir de 15 indicadores; dicha herramienta surge como respuesta de un grupo de investigadores de la Universidad Nacional Autónoma de México (UNAM), quienes realizan una crítica al modelo productivista que, con enfoque mecanicista y reduccionista, ha generado la crisis del modelo de desarrollo vigente. Proponen estos investigadores como alternativa un enfoque multidimensional de desarrollo sustentable que privilegie el bienestar social y la conservación ambiental, donde la sustentabilidad incorpore un nuevo paradigma al concepto de desarrollo (6); en consecuencia, plantean la herramienta MESMIS, que permite evaluar aspectos que tengan validez para un determinado lugar geográfico, en una escala espacial y en un período determinado, ya que la sustentabilidad no se puede determinar per se, sino a través de la comparación de un sistema transversalmente con uno de referencia, o longitudinalmente a lo largo del tiempo.

\section{Metodología}

La microcuenca del río Cormechoque tiene un área aproximada de $20 \mathrm{~km}^{2}$, entre los municipios de Soracá y Siachoque, con alturas desde los 2650 msnm hasta los $3000 \mathrm{msnm}$, temperatura entre 4 ${ }^{\circ} \mathrm{C}$ y $16{ }^{\circ} \mathrm{C}$ y precipitación promedio de $650 \mathrm{~mm} /$ año (7). Se identificaron y seleccionaron treinta fincas (agroecosistema) representativas del sistema de agricultura familiar campesina en las veredas Chaine (Soracá), Juruvita, Tocavita, Siachoque arriba y Cormechoque bajo (Siachoque). Para seleccionar las "fincas tipo" se realizó una tipificación de los agroecosistemas, siguiendo la metodología propuesta por Escobar y Berdagué (8). Como resultado de este proceso se generaron tres grupos, cada uno de los cuales tiene características homogéneas en aspectos productivos, tecnológicos, ambientales y socioeconómicos. De cada grupo se seleccionó un agroecosistema "finca tipo" para realizar la evaluación de la sustentabilidad durante el período de junio a diciembre de 2015. Para 
facilitar la identificación de cada agroecosistema evaluado, se les denominó con el nombre de la vereda a la que pertenecen (Chaine, Tocavita y Juruvita).

Para evaluar la sustentabilidad de agroecosistemas, el método MESMIS propone como primera etapa la caracterización de los agroecosistemas familiares campesinos, usando la encuesta como instrumento para capturar la información. En la caracterización se identifican un conjunto de atributos: Productividad, Estabilidad, Resiliencia, Adaptabilidad, Equidad y Autogestión, a partir de los cuales se derivan varios criterios: Retorno, Eficiencia, Conservación, Diversidad, Participación, Capacidad de Cambio e Innovación y Autosuficiencia. Para determinar el nivel de sustentabilidad de cada agroecosistema se evaluaron dichos criterios a partir del desempeño de un grupo de indicadores propuestos expresados en función de un juicio de valor sobre lo que se considera un estándar o desempeño adecuado de aspectos agropecuarios o ambientales (9). Se hace una estandarización de los indicadores, para lo cual los autores proponen una escala valorativa de $1 \mathrm{a}$ 5 , donde 1 representa el nivel de desempeño más bajo, o práctica inadecuada, y 5 , la práctica ideal o mejor condición que puede tener el indicador. Los criterios evaluados en este estudio fueron:

1. Retorno: Son los beneficios financieros que se obtienen por la inversión monetaria; está constituido por el indicador Valor Presente Neto (VPN). Para establecer la escala valorativa, se estimó el porcentaje del VPN que cubre el total de los costos de producción, teniendo como criterio que un adecuado desempeño para estos agroecosistemas es del 30 \%. La ecuación utilizada fue:

$$
V P N=\Sigma B T-\Sigma C T /(1+r)^{t}
$$

2. Eficiencia: Indica la proporción entre el retorno y la inversión monetaria. Se realizó mediante el indicador beneficio/costo (B/C). Para esta proporción se utilizó una tasa de interés del 9,47 $\%{ }^{4}(\mathrm{r})$ anual $(\mathrm{t})$, aplicada a los costos de producción (CT) y al beneficio total (BT). Para la escala valorativa (Tabla I) se consideró como ideal el valor beneficio/costo de 1,3, con el cual se recupera la inversión y se deja una utilidad ${ }^{5}$ adicional. El (B/C)

se calcula así: $B C=\left(\frac{\Sigma B T /(1+r)^{t}}{\Sigma C T /(1+r)^{t}}\right)^{1}$

3. Conservación: Indica el grado en que un agroecosistema preserva su estructura y función y la base de recursos que lo sostienen; está conformado por cuatro indicadores: cobertura vegetal, disponibilidad de agua, presión de plaga y calidad del suelo. La determinación de la cobertura vegetal se realizó mediante monitoreos quincenales en los componentes agrícola, pecuario y forestal, obteniendo un promedio ponderado de acuerdo con el área ocupada. La escala valorativa de este indicador se construye con un supuesto ideal de cobertura total del suelo (Tabla I). Para el cálculo del índice de cobertura vegetal se utilizó la siguiente expresión ${ }^{6}$ :

4 Tasa de interés promedio establecida por el Banco Agrario para préstamos a pequeños productores en el período junio-diciembre de 2015

5 Por cada 100 pesos que se invierten retornan 130 pesos al agroecosistema, es decir, se obtiene una utilidad del 30 $\%$, considerándose una actividad rentable.

6 ICVT = Índice de cobertura vegetal total; $\dot{X} \mathrm{CTA}=$ Promedio ponderado cobertura total uso de suelo agrícola;

$\dot{\mathrm{XCTP}}=$ Promedio ponderado cobertura total uso de suelo pecuario; $\dot{\mathrm{X} C T F}=$ Promedio ponderado cobertura total uso de suelo forestal; A1, 2 y 3 = Área de dedicación agrícola, pecuaria y forestal: $\mathrm{AT}=$ Área total del agroecosistema. 


$$
\begin{gathered}
I C V T=\dot{\mathrm{X}} \mathrm{CTA}+\dot{\mathrm{X} C T P}+\dot{\mathrm{X} C T F} ; \dot{\mathrm{X} C T A}=\sum \mathrm{CTA} *\left(\frac{A 1}{A T}\right) * 100 ; \dot{\mathrm{X} C T P}=\sum \mathrm{CTP} *\left(\frac{A 2}{A T}\right) * 100 ; \\
\dot{\mathrm{X} C T F}=\sum \mathrm{CTF} *\left(\frac{A 3}{A T}\right) * 100
\end{gathered}
$$

El segundo indicador de este criterio es la disponibilidad de agua/unidad de superficie y su velocidad de recarga. Este indicador pretende determinar la capacidad de almacenamiento de agua para riego y el tiempo requerido para reponer tal reserva. Se evaluó a partir de aforos quincenales durante el período de junio a diciembre de 2015 en las fuentes proveedoras (reservorios y nacimientos de agua). El índice se obtiene de promediar el indicador de la disponibilidad en litros por hectárea y la velocidad de recarga. Para tal fin se estableció como escenario ideal la escala de $250 \mathrm{~m}^{3}$ por hectárea $^{7}$ y una velocidad de recarga de 35 litros por segundo. Se calcula mediante la ecuación ${ }^{8}$ :

$$
D A=\frac{X V 1+X V 2+X V 3 \ldots \ldots X V n}{A T} ; V=W * L * H
$$

Tabla I. Escala para la cobertura vegetal y la disponibilidad de agua.

\begin{tabular}{cccc}
\hline $\begin{array}{c}\text { Nivel de } \\
\text { desempeño }\end{array}$ & $\begin{array}{c}\text { \% de } \\
\text { Cobertura } \\
\text { vegetal }\end{array}$ & $\begin{array}{c}\text { Disponibilidad } \\
\text { de agua }\left(\mathbf{m}^{3)}\right.\end{array}$ & $\begin{array}{c}\text { Capacidad } \\
\text { de recarga } \\
(\mathbf{L} / \mathbf{S})\end{array}$ \\
\hline $\mathbf{1}$ & $<10$ & $0-62$ & $0-8$ \\
$\mathbf{2}$ & $10-30$ & $62-125$ & $8-17$ \\
$\mathbf{3}$ & $30-50$ & $125-187$ & $17-25$ \\
$\mathbf{4}$ & $70-50$ & $187-250$ & $25-35$ \\
$\mathbf{5}$ & $>70$ & $>250$ & $>35$ \\
\hline
\end{tabular}

Fuente: Modificado $(13,14)$.

El tercer indicador es la presión de insectos plaga y de enfermedades en el agroecosistema; para su evaluación se diseñó una metodología de monitoreo con lecturas periódicas durante el ciclo de cultivo para los principales agentes biológicos. La afectación se obtuvo con la ecuación ${ }^{9}$ :

7 Para mantener una producción anual estable se requieren aproximadamente $250 \mathrm{~m} 3 / \mathrm{ha} \mathrm{(10)}$.

$8 \quad \mathrm{DA}=$ Disponibilidad de agua; $\mathrm{XVn}=$ Promedio volumen de agua fuentes; $V=$ Volumen; $W=$ ancho de la fuente.

$\mathrm{L}=$ Largo de la fuente; $\mathrm{H}=$ Profundidad; $\mathrm{AT}=$ Área total.

$9 \mathrm{PPE}=$ Presión de plagas y enfermedades; $\mathrm{Xpn}=$ Promedio de incidencia de plaga o enfermedad; $X m n=$ Promedio
$P P E=\frac{X p 1+X p 2+X p 3 \ldots \ldots n}{n} ; X p n=\frac{X m 1+X m 2+X m 3 \ldots . n}{n}$

La escala valorativa del indicador se estableció teniendo como referencia el umbral de daño económico para cada uno de los agentes biológicos identificados, donde el valor 1 corresponde a un nivel de infestación alto, y 5, a un nivel de infestación bajo. Se monitorearon las principales plagas y enfermedades ${ }^{10}$ del cultivo de papa (16, 17).

El indicadorcalidad del suelo evalúa las propiedades físicas y químicas. La escala valorativa (siendo 5 la mejor condición posible, y 1 la menos deseable o limitante para la actividad agropecuaria) toma como referentes la concentración o propiedades óptimas, así, en la medida que estas descienden, el desempeño del indicador es menor (18). Para su cálculo se usó la siguiente ecuación ${ }^{11}$ :

$$
\mathrm{X}=\frac{\mathrm{I} 1+\mathrm{I} 2++\mathrm{I} 3+\cdots \mathrm{ln}}{\mathrm{n}}
$$

4. Diversidad: Indica la riqueza de elementos que tiene un agroecosistema en un momento determinado. Está constituida por dos indicadores: biodiversidad agropecuaria y uso de semillas locales; el primero se mide teniendo en cuenta la riqueza específica (número de especies del agroecosistema) y la equitabilidad (distribución de la abundancia de las especies). Se usó el índice de Shannon (19), que asume que los individuos son seleccionados al azar y que todas las especies están representadas en la muestra. Adquiere un valor de cero (0) cuando está presente una única especie y cinco (5) cuando la diversidad es mayor

de incidencia de plagas por lectura de registro; $n=$ Número de muestras o lecturas.

10 Considerado como ataque que causa una pérdida económica afectando la rentabilidad del cultivo.

11 Donde: $X=$ Indicador calidad del suelo; $I n=$ Valor indicador por característica física o química $\mathrm{n}=$ Parámetros evaluados. 
(20). Para determinar el índice se usó la siguiente fórmula (21):

$$
H=\sum_{i=1} s(p i)\left(\log _{2} p i\right)
$$

El indicador uso de semillas locales (ISL) se obtiene mediante el cociente entre el uso total de semillas locales (SL) sobre la compra de semilla comercial (SC) (9). La escala valorativa construida para determinar el comportamiento del indicar presupone como condición ideal el uso de más del $70 \%$ de semillas locales (Tabla II).

5. Participación: Refiere el grado en que los actores sociales y personas participan en actividades de desarrollo. Para este criterio se evaluaron dos indicadores: el primero es el empleo generado, que permite determinar la cantidad de empleos familiares que demanda el agroecosistema por ciclo cultivo; la escala valorativa se estableció tomando como referencia que el $60 \%{ }^{12}$ de los empleos se provean con mano de obra familiar. Para el cálculo de este indicador se usó la siguiente ecuación ${ }^{13}$ :

$E E=\frac{T E(\% E E)}{100 \%} ; E F C=T E-E E ; \% E F=\frac{\sum E F C * 100}{T E}$

Tabla II. Escala para uso de semillas, mano de obra y participación.

\begin{tabular}{cccc}
\hline $\begin{array}{c}\text { Nivel de } \\
\text { desempeño }\end{array}$ & $\begin{array}{c}\text { Uso de } \\
\text { semillas } \\
\text { locales } \\
\mathbf{( \% )}\end{array}$ & $\begin{array}{c}\text { Mano } \\
\text { de obra } \\
\text { familiar } \\
\mathbf{( \% )}\end{array}$ & $\begin{array}{c}\text { Participación } \\
\text { en asociaciones } \\
\text { locales (\%) }\end{array}$ \\
\hline $\mathbf{1}$ & $<5$ & $<20$ & $<20$ \\
$\mathbf{2}$ & $5-20$ & $20-40$ & $20-40$ \\
$\mathbf{3}$ & $20-50$ & $40-60$ & $40-60$ \\
$\mathbf{4}$ & $50-70$ & $60-80$ & $60-80$ \\
$\mathbf{5}$ & $>70$ & $>80$ & $80-100$ \\
\hline
\end{tabular}

Fuente: La investigación.

12 Arbitrariamente se estableció este valor por considerarlo representativo de la cantidad de mano de obra que en condiciones de la zona de estudio es proveído por la familia rural.

13 Donde: $\mathrm{EE}=$ Empleo externo, $\mathrm{TE}=$ Total de empleos; $\mathrm{EFC}=$ Empleo familiar cultivo; $\% \mathrm{EF}=$ Porcentaje de empleo familiar.
El segundo indicador de este criterio es participación en asociaciones locales. Se identificaron las asociaciones existentes a nivel local y en cuáles participan los integrantes del núcleo familiar. Para la escala valorativa el referente ideal es participar en más del $70 \%$ de las organizaciones existentes (Tabla II).

6. Capacidad de cambio e innovación: Este criterio está conformado por dos indicadores: Capacidad de innovación tecnológica y Capacitación y generación de conocimiento. El primer indicador evalúa la apropiación de tecnología y prácticas agrícolas innovadoras en el agroecosistema; se propuso como condición deseable siete o más prácticas innovadoras en los últimos cinco años; se determina mediante ${ }^{14}$ :

$$
C I T=\sum P
$$

El segundo indicador se determinó mediante la cuantificación de asistencia de integrantes del núcleo familiar a actividades de capacitación en el último año (Tabla III).

7. Autosuficiencia: Está conformado por tres indicadores: Dependencia de insumos externos (DIE), Ahorro interno (Al) y Alimentos de la canasta familiar provenientes del agroecosistema (API). El primer indicador se estimó estableciendo el costo de los insumos agrícolas y el porcentaje de origen externo; se obtiene del cociente entre costos totales de insumos (CTi) y el costo de insumos externos por ciclo de cultivo (Cie):

$$
\operatorname{DIE}(\%)=\left(\frac{C i e}{C T i}\right) \times 100
$$

El segundo indicador, capacidad de ahorro interno (Al), evalúa los costos de producción que son cubiertos con préstamos (fuentes externas de financiación); para tal fin se calculó el cociente entre el costo total (CT) de producción y el costo total cubierto con préstamo (CTP); se calcula mediante: (22):

$14 \mathrm{CIT}=$ Capacidad de innovación tecnológica; $\mathrm{P}=$ Prácticas de innovación tecnológica. 


$$
A I=C T / C T P ; C T P=\% C T P * C T
$$

El tercer indicador, alimentos que se proveen con productos internos (API), expresado en pesos (\$), se determinó a partir del costo total de alimentación de la familia, identificando el porcentaje de alimentos proveniente del propio agroecosistema. El coeficiente se obtiene entre el costo total de alimentos (CTA) y el costo de alimentos que provee la producción interna del agroecosistema (CAi). La escala valorativa propone como desempeño adecuado cuando el $60 \%{ }^{18}$ del costo de los alimentos provienen del mismo agroecosistema (23). Se calcula mediante:

$$
A P I=\left(\frac{C T A}{C A i}\right) * 100
$$

Tabla III. Escala para la capacidad de cambio y la autosuficiencia.

\begin{tabular}{cccccc}
\hline $\begin{array}{c}\text { Nivel de } \\
\text { desempeño }\end{array}$ & $\begin{array}{c}\text { Innovación } \\
\text { tecnológica }\end{array}$ & $\begin{array}{c}\text { Capacitación en } \\
\text { eventos }\end{array}$ & $\begin{array}{c}\text { Dependencia de } \\
\text { insumos externos } \\
(\%)\end{array}$ & $\begin{array}{c}\text { Ahorro } \\
\text { interno } \\
\text { préstamo \% }\end{array}$ & $\begin{array}{l}\text { Alimentos } \\
\text { p r o d u c c i ó n } \\
\text { interna (\%) }\end{array}$ \\
\hline $\mathbf{1}$ & $<1$ & $1-3$ & $>80$ & $>80$ & $<20$ \\
\hline $\mathbf{3}$ & $1-3$ & $3-5$ & $80-60$ & $80-60$ & $20-30$ \\
$\mathbf{4}$ & $3-5$ & $5-7$ & $60-40$ & $60-40$ & $30-40$ \\
$\mathbf{5}$ & $5-7$ & $7-10$ & $40-20$ & $40-20$ & $40-60$ \\
\hline
\end{tabular}

Fuente: La investigación (a partir de consensos con los agricultores).

\section{Resultados y discusión}

Los resultados del análisis comparativo del comportamiento de los indicadores para determinar los niveles de sustentabilidad en los tres agroecosistemas (9) se presentan gráficamente mediante la construcción de mapas tipo radar que muestran el comportamiento del conjunto de criterios evaluados (cada uno conformado por indicadores).

La caracterización de los tres agroecosistemas campesinos (Tocavita, Chaine y Juruvita) se determinó a partir del análisis comparativo de sus componentes biofísicos, bióticos, tecnológicos y socioeconómicos; en la Tabla IV se presentan los resultados. En el componente socioeconómico se encuentran amplias diferencias entre los agroecosistemas; la primera es el porcentaje de aporte de mano de obra familiar para las actividades agropecuarias, que va desde únicamente el $20 \%$, en Juruvita, hasta el $70 \%$, en Chaine. Igualmente, la canasta familiar se provee en cantidades distintas de producción interna, desde el $70 \%$, en Chaine, hasta el $40 \%$, en Juruvita, lo cual reviste interés, ya que muestra el grado de exposición de estas familias a factores externos de mercado y de inseguridad alimentaria.

$15 \quad$ El nivel de desempeño en la escala valorativa de 1 (total dependencia de alimentos externos) a 5 (Desempeño ideal), donde el consumo de alimentos que provengan de la finca sea superior al $60 \%$, ya que no toda la canasta familiar es posible que se produzca en el agroecosistema. 
Tabla IV. Caracterización de agroecosistemas familiares campesinos.

\begin{tabular}{|c|c|c|c|c|}
\hline \multicolumn{2}{|r|}{ Características/ agroecosistema } & Chaine & Juruvita & Tocavita \\
\hline \multirow{5}{*}{$\frac{\pi}{\frac{\pi}{0}}$} & Altura & 2920 msnm & $2850 \mathrm{msnm}$ & $2890 \mathrm{msnm}$ \\
\hline & Precipitación media & & 650 mm/año & \\
\hline & Pendiente área agrícola & $30-40 \%$ & $20-40 \%$ & $20-30 \%$ \\
\hline & Pendiente área pecuaria & $>40$ & $20-30 \%$ & $40-50 \%$ \\
\hline & Tipo de suelo & Franco arenoso & Franco arcilloso & Franco arcilloso \\
\hline \multirow{5}{*}{ 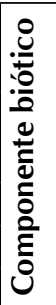 } & Tipo de explotación & Agrícola, pecuaria & Agrícola y pecuaria & $\begin{array}{c}\text { Agrícola, pecuario y } \\
\text { silvopastoril }\end{array}$ \\
\hline & Cultivos de interés económico & $\begin{array}{l}\text { Papa, zanahoria, maíz, } \\
\text { cereales/ 3,8 h }\end{array}$ & $\begin{array}{c}\text { Papa, zanahoria, maíz, } \\
\text { mora/ } 3,0 \mathrm{~h}\end{array}$ & $\begin{array}{l}\text { Papa, arveja, maíz, } \\
\text { mora, cereales/ 3,1 h }\end{array}$ \\
\hline & Especies de interés forestales & 11 & 4 & 6 \\
\hline & Especies Animales de interés & 4 & 3 & 4 \\
\hline & Tipo de bosque & Nativo, reforestación, & Reforestación & Nativo, reforestación \\
\hline
\end{tabular}

\begin{tabular}{|c|c|c|c|c|}
\hline \multirow{7}{*}{ 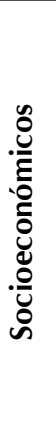 } & Área del agroecosistema & 6,37 ha & 4,84 ha & 5,5 ha \\
\hline & Integrantes núcleo familiar & 7 & 6 & 5 \\
\hline & Dedicación del núcleo familiar & $85 \%$ dedicado & $33 \%$ dedicado & $80 \%$ dedicado \\
\hline & como mano de obra & exclusivamente & exclusivamente & exclusivamente \\
\hline & Mano de obra agrícola & $70 \%$ familiar & $20 \%$ familiar & $70 \%$ familiar \\
\hline & $\begin{array}{l}\text { Destino de la producción } \\
\text { agrícola }\end{array}$ & $75 \%$ venta & $80 \%$ venta & $80 \%$ venta \\
\hline & Comercialización agrícola & $\begin{array}{c}100 \% \text { plazas de } \\
\text { mercado }\end{array}$ & $\begin{array}{l}95 \% \text { plazas de } \\
\text { mercado y } 5 \% \\
\text { intermediario }\end{array}$ & $\begin{array}{c}90 \% \text { plazas de mercado } \\
\text { y } 10 \% \text { intermediario }\end{array}$ \\
\hline
\end{tabular}

\begin{tabular}{|c|c|c|c|c|}
\hline \multirow{5}{*}{ 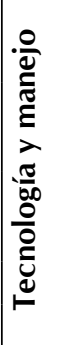 } & Arreglos productivos & $\begin{array}{l}\text { Intercalados, franjas, } \\
\text { relevo, barreras vivas }\end{array}$ & $\begin{array}{l}\text { Intercalados, relevo, } \\
\text { franjas }\end{array}$ & Intercalado, relevo \\
\hline & Preparación del suelo & $\begin{array}{c}\text { Uso de herramientas } \\
\text { conservacionistas }\end{array}$ & $\begin{array}{c}\text { Uso de herramientas } \\
\text { convencionales }\end{array}$ & $\begin{array}{l}\text { Uso de herramientas } \\
\text { conservacionistas }\end{array}$ \\
\hline & Tipos de tracción & $\begin{array}{c}\text { Mayor uso de tracción } \\
\text { humana }\end{array}$ & $\begin{array}{c}\text { Mayor uso de tracción } \\
\text { mecánica }\end{array}$ & $\begin{array}{c}\text { Mayor uso de tracción } \\
\text { humana }\end{array}$ \\
\hline & Uso de abonos orgánicos & Compost y biopreparados & Abonos verdes & Compost y bocashi \\
\hline & Control de plagas & Químico y cultural & Químico & Químico y cultural \\
\hline
\end{tabular}

Para determinar los atributos de sustentabilidad propuestos por Astier et al. (9), MESMIS identifica y evalúa los criterios de diagnóstico anteriormente mencionado; tales indicadores dan cuenta del estado de sustentabilidad. Para el criterio Retorno se evalúo como indicador el Valor Presente Neto (VPN), usando una tasa de interés de 9,47 \% anual, expresando en porcentaje respecto de los costos totales y el beneficio total del agroecosistema.
Los resultados muestran que el agroecosistema Tocavita presenta un VPN de $30 \%$, que supone una adecuada rentabilidad del ejercicio agropecuario. Este positivo desempeño se debe en buena medida a los bajos costos de producción y a los mejores precios de sus productos. Por el contrario, los agroecosistemas Chaine y Juruvita tienen un VPN inferior al $4 \%$, generando baja rentabilidad (Tabla V). 
Tabla V. Resultados indicador valor presente neto y beneficio/costo.

\begin{tabular}{|c|c|c|c|c|c|c|c|}
\hline & $\begin{array}{c}\text { Costos totales } \\
(\mathrm{CT}) \\
\end{array}$ & $\begin{array}{c}\text { Beneficios } \\
\text { totales (BT) }\end{array}$ & VPN (BT- CT) & $\begin{array}{l}\text { VPN } \\
(\%)\end{array}$ & $\begin{array}{c}\text { Desempeño } \\
\text { VPN }\end{array}$ & $\mathrm{B} / \mathrm{C}$ & $\begin{array}{c}\text { Desempeño } \\
\text { B/C }\end{array}$ \\
\hline Chaine & $\$ 33.025 .358$ & $\$ 34.457 .249$ & 1.431 .936 & 4 & 1 & 1,05 & 2 \\
\hline Juruvita & $\$ 12.721 .463$ & $\$ 13.065 .750$ & 344.287 & 3 & 1 & 1,03 & 2 \\
\hline Tocavita & $\$ 20.340 .000$ & $\$ 26.503 .600$ & 6.163 .600 & 30 & 5 & 1,3 & 4 \\
\hline
\end{tabular}

Para el criterio Eficiencia, la Tabla $\mathrm{V}$ también muestra el resultado de la relación Beneficio/Costo $(\mathrm{B} / \mathrm{C})$, donde Tocavita tiene un valor de 1,3; los demás agroecosistemas presentan un resultado ligeramente superior a 1,0 , indicando que persiste la baja rentabilidad para las actividades agropecuarias. Lo anterior muestra la difícil situación financiera y de liquidez, que coadyuva a la insatisfacción de necesidades básicas y limita las posibilidades de optimización y actualización tecnológica, así como la permanencia de la familia en el espacio rural dedicada a actividades agropecuarias.

En el criterio Conservación se evaluó el índice de cobertura vegetal para cada uso del suelo. La Tabla
VI presenta los resultados de cada agroecosistema, evidenciando amplias diferencias en la cobertura del suelo de uso agrícola, que fluctúa entre el 39 $\%$, en Juruvita, hasta el $71 \%$, en Tocavita. Estos resultados reflejan los posibles efectos sobre el suelo de las prácticas agrícolas, ya que este último agroecosistema hace mayor rotación de cultivos, tiene cultivos intercalados, de relevo e incorpora frecuentemente residuos de cosecha. Las áreas de bosque en los tres agroecosistemas tienen porcentaje de cobertura similar, pero con amplias diferencias en su tamaño. Tocavita presenta el mejor desempeño en el índice de cobertura, con un $82 \%$ de promedio ponderado, seguido de Chaine y Juruvita, con $62 \%$ y $60 \%$, respectivamente (Tabla $\mathrm{VI})$.

Tabla VI. Resultado indicador índice de cobertura.

\begin{tabular}{ccccccccc}
\hline & $\begin{array}{c}\text { Área } \\
\text { agrícola } \\
\text { (ha) }\end{array}$ & $\begin{array}{c}\text { Cobertura } \\
\text { agrícola (\%) }\end{array}$ & $\begin{array}{c}\text { Área } \\
\text { pecuaria } \\
\text { (ha) }\end{array}$ & $\begin{array}{c}\text { Cobertura } \\
\text { pecuaria } \\
(\%)\end{array}$ & $\begin{array}{c}\text { Área } \\
\text { bosque (ha) }\end{array}$ & $\begin{array}{c}\text { Cobertura } \\
\text { bosque (\%) }\end{array}$ & $\begin{array}{c}\text { Promedio } \\
\text { ponderado } \\
(\dot{X})\end{array}$ & $\begin{array}{c}\text { Desempeño } \\
\text { cobertura }\end{array}$ \\
\hline Chaine & 3,85 & 43,0 & 2,02 & 92,0 & 0,5 & 88,0 & 62,0 & 4 \\
Juruvita & 3,0 & 39,0 & 1,8 & 94,0 & 0,04 & 76,0 & 60,0 & 4 \\
Tocavita & 3,1 & 71,0 & 1,4 & 98,0 & 1,0 & 91,0 & 82,0 & 5 \\
\hline
\end{tabular}

Para el indicador Volumen de agua disponible por unidad de superficie, los resultados muestran que Chaine tiene la mayor capacidad de almacenamiento, con $207 \mathrm{~m}^{3} / \mathrm{h}$, y el tiempo promedio de recarga de los acuíferos es de 25,4 días. Tocavita tiene una infraestructura de tres reservorios y un "nacimiento"; su capacidad de almacenamiento es de $170,9 \mathrm{~m}^{3} / \mathrm{h}$ y su capacidad de recarga es de 0,53 litros por segundo, por lo que se requerirá de 14,5 días para Ilenarlos completamente. Por el contrario, Juruvita tiene menor capacidad de almacenamiento $\left(0.5 \mathrm{~m}^{3} / \mathrm{h}\right)$ y un tiempo promedio de recarga de 1,8 días. Se evidencia dificultad en estos dos últimos agroecosistemas para mantener la oferta hídrica a los cultivos, limitante que se reflejará en otros indicadores ${ }^{16}$ (Tabla VII).

16 Toma como referencia un óptimo de $250 \mathrm{~m} 3 / \mathrm{h} / \mathrm{semana}$ y capacidad de recarga de 0,6 litros/segundo, que permite mantener su reserva. 
Tabla VII. Indicadores disponibilidad de agua y velocidad de recarga.

\begin{tabular}{cccccc}
\hline & $\begin{array}{c}\text { Disponibilidad } \\
\text { de agua }\left(\mathbf{m}^{\mathbf{3}} \mathbf{h}\right)\end{array}$ & $\begin{array}{c}\text { Escala de } \\
\text { desempeño }\end{array}$ & $\begin{array}{c}\text { Recarga litro/ } \\
\mathbf{s e g}\end{array}$ & $\begin{array}{c}\text { Escala de } \\
\text { desempeño }\end{array}$ & $\begin{array}{c}\text { Promedio } \\
\text { escala de } \\
\text { desempeño }\end{array}$ \\
\hline Tocavita & 170,9 & 3 & 0,75 & 5 & 4,0 \\
Chaine & 207 & 4 & 0,60 & 4 & 3,5 \\
\hline Juruvita & 0,53 & 1 & 0,016 & 1 & 1,0 \\
\hline
\end{tabular}

Fuente: la investigación.

El resultado del indicador Presión de plagas y enfermedades muestra alta incidencia en los tres agroecosistemas; sin embargo, se destaca que en Tocavita la presión de este indicador tiene un valor de 3,5 en la escala valorativa, mientras que en Juruvita y Chaine es de 4,2 y 4,6, respectivamente. La Figura 1 muestra el radar comparativo de la incidencia de plagas y enfermedades en los tres agroecosistemas; en cada eje del radar se muestra el comportamiento del agente biológico en la escala propuesta (de 1 a 5). Se evidencia que el mejor comportamiento lo tienen los agroecosistemas Chaine y Juruvita. Por su parte, Tocavita tiene valores intermedios (de la escala), que supone una mejor disposición para albergar diversidad biológica en los cultivos.

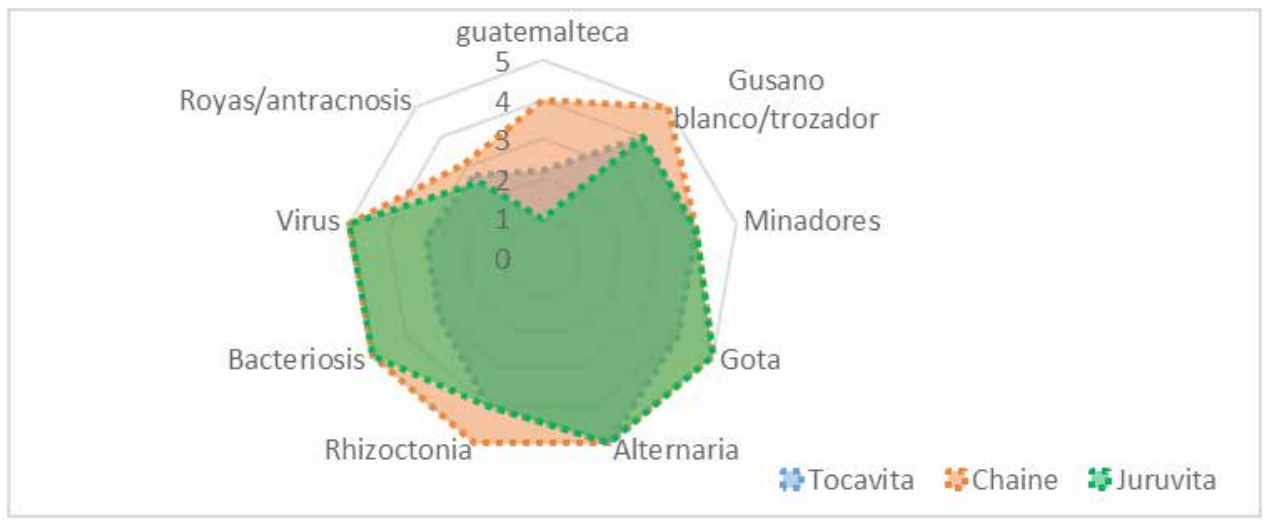

Figura 1. Radar comparativo incidencia de plagas y enfermedades.

Para el indicador Calidad del suelo, los parámetros del análisis se expresaron usando la escala valorativa propuesta: entre 1 (condición menos deseable) y 5 (nivel de óptima disponibilidad de un elemento nutricional). Los agroecosistemas Juruvita y Chaine presentan los niveles más bajos de materia orgánica (M.O.), calcio y magnesio, y Tocavita presenta adecuados niveles de M.O., bases cambiables y elementos menores, excepto cobre, que es deficitario en todos los agroecosistemas (Figura 2). 


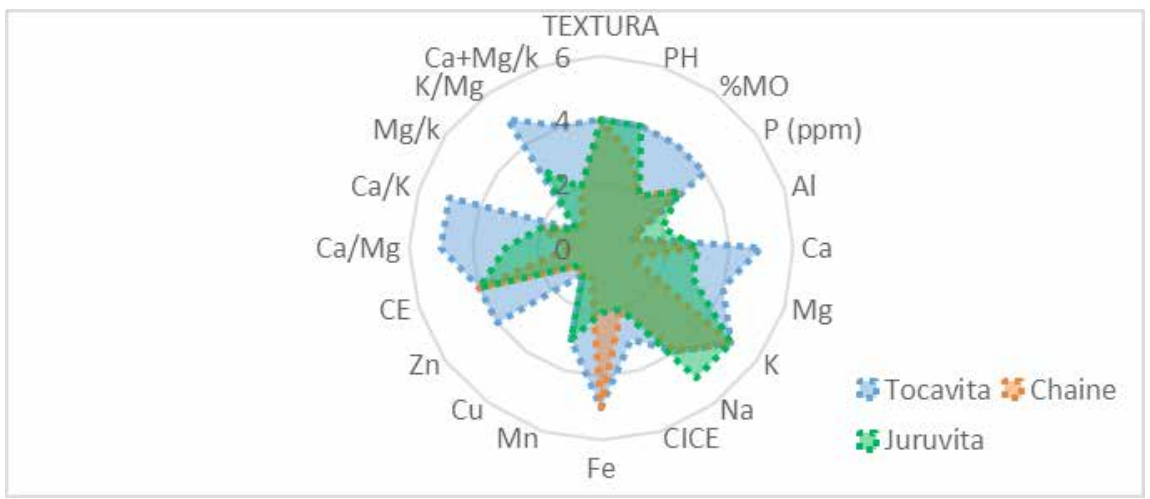

Figura 2. Comparación indicador Calidad del suelo.

El criterio Diversidad evaluó los indicadores Biodiversidad agropecuaria e Índice de uso de semillas locales. Los resultados muestran que para el primer indicador, valorado mediante el índice de Shannon (1985), Juruvita tiene el índice más bajo $(1,0)$, denotando baja diversidad de especies y poca equidad entre ellas, con tendencia al monocultivo, que se evidencia en la escasez de especies nativas con funciones de protección, seguridad alimentaria, medicinales o como fuentes energéticas. Tocavita tiene un índice de 2,55, que indica mejor riqueza específica y abundancia relativa. Este agroecosistema tiene mayor cantidad de especies que cumplen funciones de seguridad alimentaria, medicinales, protectoras de suelo y fuentes de agua, entre otras (Tabla VIII).

Tabla VIII. Resultados Diversidad, participación e innovación.

\begin{tabular}{|c|c|c|c|}
\hline & Tocavita & Chaine & Juruvita \\
\hline \multicolumn{4}{|c|}{ Criterio diversidad } \\
\hline \multicolumn{4}{|c|}{ Índice de Shannon } \\
\hline $\begin{array}{l}\text { Cantidad de } \\
\text { especies }\end{array}$ & 279517 & 203649 & 162755 \\
\hline Índice & 2,55 & 1,79 & 1,0 \\
\hline $\begin{array}{l}\text { Escala de } \\
\text { desempeño }\end{array}$ & 3 & 2 & 1 \\
\hline \multicolumn{4}{|c|}{ Indicador Uso de semillas locales } \\
\hline Semilla Local (\%) & 60 & 50 & 67 \\
\hline $\begin{array}{l}\text { Índice uso de } \\
\text { semillas locales }\end{array}$ & 1,5 & 1,0 & 2,0 \\
\hline $\begin{array}{l}\text { Escala de } \\
\text { desempeño }\end{array}$ & 4 & 3 & 4 \\
\hline \multicolumn{4}{|c|}{ Criterio participación } \\
\hline \multicolumn{4}{|c|}{ Indicador Empleo generado } \\
\hline $\begin{array}{l}\text { Total } \\
\text { empleos } \\
\text { generados }\end{array}$ & 857 & 803 & 207 \\
\hline Empleo Familiar & 683 & 618 & 152 \\
\hline (\%) Empleo & 80 & 77 & 74 \\
\hline Familiar & & & \\
\hline $\begin{array}{l}\text { Escala de } \\
\text { desempeño }\end{array}$ & 5 & 4 & 4 \\
\hline
\end{tabular}




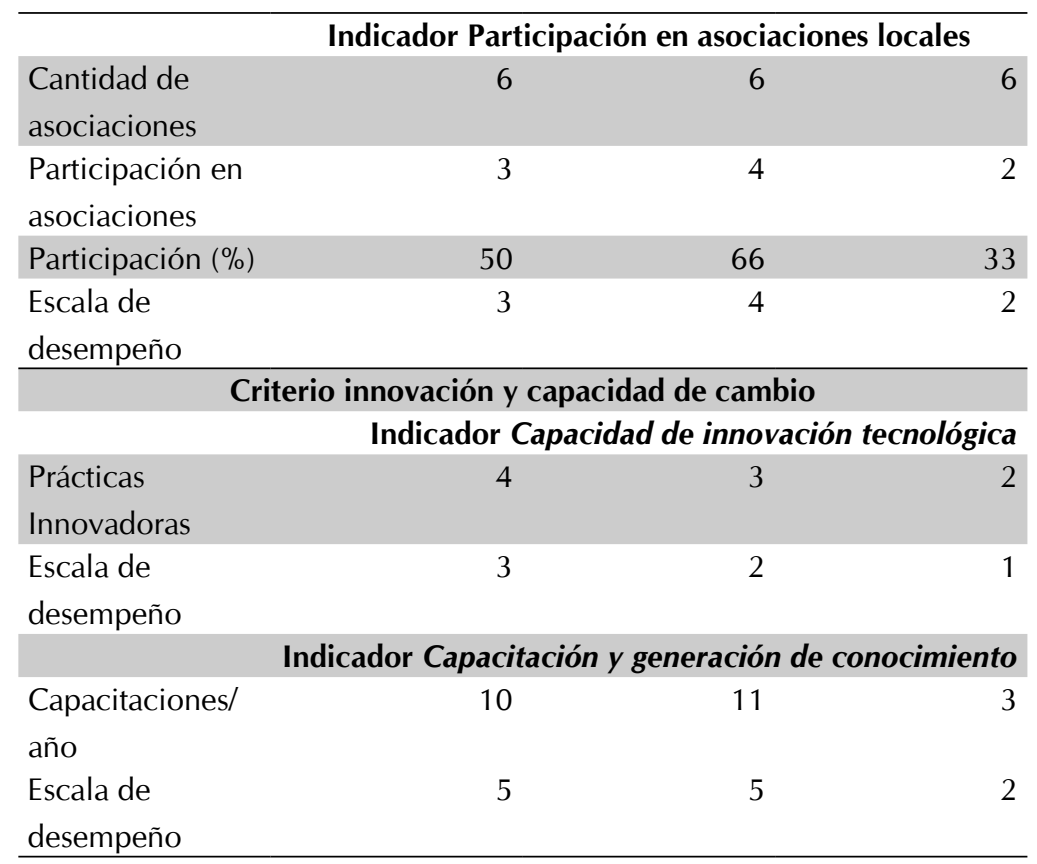

Fuente: La investigación.

Para el segundo indicador, Índice de uso de semillas locales, se evaluó el porcentaje de semillas locales versus las adquiridas comercialmente. Los resultados muestran que Juruvita tiene el porcentaje más alto en el uso de semillas locales, con $67 \%$, seguida de Tocavita, con $60 \%$, y Chaine, con 50 $\%$.

El criterio Participación evalúa dos indicadores: empleo generado y participación en organizaciones sociales. En el primero se determinó que Chaine y Tocavita generan mayor cantidad de empleos (Tabla VIII), de los cuales, el $77 \%$ y el $80 \%$, respectivamente, son proveídos por la familia campesina, mientras que Juruvita genera menos empleos, por su menor dinámica productiva, pero el porcentaje que provee la familiar es similar a los demás agroecosistemas (74\%). Esto implica que la migración de integrantes del núcleo familiar posiblemente ha estado relacionada con su reducida generación de empleo ${ }^{17}$.

17 Se consideró arbitrariamente el valor ideal de 60 $\%$ de mano de obra familiar y un $40 \%$ de mano de obra externa, ya que, por el área, los agroecosistemas demandan mayor cantidad de jornales que los disponibles en el núcleo familiar, dando oportunidad laboral en la región.
El criterio Innovación y capacidad de cambio tiene dos indicadores: Capacidad de innovación tecnológica y Capacitación y generación de conocimiento en los últimos cinco años. En el primero se identificó que los tres agroecosistemas evaluados presentan baja capacidad de innovación, reflejada en la escasa cantidad de nuevas prácticas agrícolas. El agroecosistema Tocavita tiene el mejor desempeño, con 4 prácticas innovadoras en: protección de suelos, conservación de agua, almacenamiento de semillas y manejo fitosanitario. Chaine y Juruvita tienen, respectivamente, 3 y 2 prácticas innovadoras.

El segundo indicador muestra que los agricultores de Chaine y Tocavita asisten a mayor cantidad de eventos de capacitación, lo que sugiere la posible relación entre este aspecto y la cantidad de innovaciones tecnológicas que se implementan en el ejercicio agropecuario. En tal sentido, Juruvita muestra un rezago en su intención de capacitación que, a su vez, se refleja en la cantidad de prácticas innovadoras que desarrolla (Tabla IX).

El criterio Autosuficiencia está constituido por tres indicadores: 1) Dependencia de insumos externos, 2) Capacidad de ahorro y 3) Alimento que se 
provee con producción interna. Para el primer indicador los resultados evidencian que existe alta dependencia de insumos externos en los tres agroecosistemas, principalmente por compra de semilla, fertilizantes y productos fitosanitarios; en este indicador se destaca el desempeño de Tocavita, que presenta el menor porcentaje de dependencia $(67,1 \%)$, ya que usa abonos de fuentes orgánicas e implementa diversas herramientas para el manejo fitosanitario de los cultivos; al contrario, Juruvita presenta una difícil condición, pues ha venido perdiendo su autosuficiencia, manifiesta en el alto porcentaje que representa la compra de insumos respecto del total de sus costos $(85.5 \%)$, lo cual limita su capacidad financiera en las actividades agropecuarias, disminuyendo su posibilidad de transformación e innovación (Tabla IX).
En el segundo indicador, Capacidad de ahorro interno, Juruvita presenta el mejor desempeño, con un índice de ahorro interno de 3.71, es decir, que cerca del $27 \%$ de los costos de producción provienen de crédito externo; mientras que Tocavita y Chaine requieren el $40.3 \%$ y el $71 \%$, respectivamente. Lo anterior explica las dificultades financieras de estos dos agroecosistemas para el desarrollo de las actividades agropecuarias. El resultado de Juruvita también está influenciado por su limitada actividad agropecuaria, convirtiéndose en un indicador que muestra el descenso de esta actividad por el aumento de integrantes de la familia que se desplazan a actividades no agropecuarias (Tabla IX).

Tabla IX. Resultados indicadores del criterio Autosuficiencia.

\begin{tabular}{|c|c|c|c|}
\hline & Tocavita & Chaine & Juruvita \\
\hline \multicolumn{4}{|c|}{ Indicador dependencia de insumos } \\
\hline Costo total insumos $(\$)$ & 18.396.100 & 11.744 .236 & 3.298 .559 \\
\hline $\begin{array}{l}\text { Costo insumos externos } \\
(\$)\end{array}$ & 12.351 .690 & 8.748 .438 & 2.820 .756 \\
\hline $\begin{array}{l}\text { Dependencia insumos } \\
\text { externos (\%) }\end{array}$ & 67,1 & 74,4 & 85,5 \\
\hline Escala de desempeño & 2 & 2 & 1 \\
\hline \multicolumn{4}{|c|}{ Indicador capacidad de ahorro interno } \\
\hline $\begin{array}{l}\text { Costo total de } \\
\text { producción \$ (CT) }\end{array}$ & 34.042 .634 & 25.972 .860 & 10.997 .153 \\
\hline $\begin{array}{l}\text { Costo cubierto con } \\
\text { préstamos } \$(\mathrm{CP})\end{array}$ & 13.729 .569 & 18.397 .666 & 2.962 .164 \\
\hline $\begin{array}{l}\text { Costos cubiertos con } \\
\text { préstamo }(\%)\end{array}$ & 37 & 70,8 & 26,9 \\
\hline Escala de desempeño & 4 & 2 & 4 \\
\hline \multicolumn{4}{|c|}{ Indicador alimento que se provee con la producción interna } \\
\hline $\begin{array}{l}\text { Costo de alimentos que } \\
\text { consume la familia / } \\
\text { mes (CTA) \$ }\end{array}$ & 500.000 & 600.000 & 550.000 \\
\hline $\begin{array}{l}\text { Costo de alimentos que } \\
\text { provienen de la finca } \\
\text { (CAF) \$ }\end{array}$ & 250.000 & 230.000 & 115.000 \\
\hline $\begin{array}{l}\text { Costo de alimentos } \\
\text { provenientes de la finca } \\
(\%)\end{array}$ & 50 & 38,3 & 21 \\
\hline Escala de desempeño & 4 & 3 & 2 \\
\hline
\end{tabular}

Fuente: La investigación. 
En el análisis integrado del agroecosistema Juruvita se evidencia un bajo nivel de desempeño en los indicadores de seguridad alimentaria y capacidad de capitalización, implicando por tanto mayor vulnerabilidad a condiciones adversas de tipo financiero y de mercado. Los resultados hacen evidente la baja oferta ambiental, especialmente en indicadores que son soporte del agroecosistema, como la disponibilidad de agua y la diversidad de especies (índice de Shannon). Lo anterior permite inferir la posible relación entre los bajos niveles de diversidad con la disponibilidad de agua, manifestándose también en el bajo desempeño del indicador de calidad del suelo, que a su vez repercute en la producción de alimentos y la dependencia de insumos externos, afectando los resultados de los indicadores de tipo financiero. De la misma forma, el porcentaje de cobertura vegetal está directamente influenciado por la mayor cantidad de área dedicada a pradera con respecto al área agrícola.

El agroecosistema Chaine presenta bajo nivel de desempeño en los indicadores financieros, como baja capacidad de ahorro interno, alta dependencia de crédito y constante descapitalización, que imposibilitan generar innovaciones productivas y tecnológicas. Lo anterior, posiblemente, explica las condiciones adversas que en general tiene el sistema de producción campesina, evidenciando riesgo para su permanencia. El desempeño óptimo del indicador Presión de plagas y enfermedades indica un adecuado manejo fitosanitario del cultivo que puede estar relacionado inversamente con el alto índice de dependencia de insumos externos y la frecuente aplicación de agroquímicos para su control. Los indicadores que en buena medida soportan la permanencia de este agroecosistema son el porcentaje de alimentos que provee la producción interna y la generación de empleo cubierto por mano de obra familiar, los cuales mantienen la cohesión del núcleo familiar alrededor de actividades agropecuarias, contrario a las condiciones de Juruvita, cuyo núcleo se ha fragmentado por las condiciones de baja rentabilidad e insuficiente oferta de empleo durante el año, que presionó a varios miembros de la familia rural a buscar alternativas laborales en otros municipios y en actividades no agropecuarias.

El agroecosistema Tocavita presenta los mejores niveles de desempeño en los indicadores financieros, reflejado en una mejor condición de autosuficiencia y capacidad de permanencia en el tiempo; posiblemente, estos resultados se explican por un buen desempeño de los indicadores de cobertura vegetal, uso de semillas locales y oferta ambiental (disponibilidad de agua, calidad de suelos, diversidad de especies, etc.) entre otros. Lo anterior permite inferir que la sustentabilidad de este agroecosistema se basa en la ejecución de prácticas agropecuarias conservacionistas, gestión de fuentes de agua, capacitación e innovación constante de todos los integrantes del núcleo familiar y uso de tecnologías de bajo costo y bajo riesgo, entre otras.

Para evidenciar el nivel de sustentabilidad se contrastan los resultados de los indicadores evaluados, encontrando amplias diferencias en su comportamiento en cada agroecosistema y entre los tres agroecosistemas. En la Figura 3 y la Tabla X se presenta el análisis integrado de los indicadores evaluados. Se evidencia que Juruvita tiene el más bajo nivel de sustentabilidad; esta condición se puede explicar por su escasa diversidad y disponibilidad de agua, así como por el bajo desempeño en los indicadores capacidad tecnológica y participación en organizaciones sociales, que afectan su dinámica productiva y social. Tal condición se refleja en el desempeño de los indicadores que dan cuenta de la rentabilidad, así como de su alta dependencia de factores externos. En igual sentido, Tocavita tiene el nivel de sustentabilidad más alto entre los agroecosistemas evaluados, gracias al desempeño del grupo de indicadores de oferta ambiental, el cual se refleja en mejor desempeño de los financieros, superior al $80 \%$ en la escala propuesta entre 1 y 5; por el contrario, Chaine y Juruvita presentan valores cercanos al $20 \%$, denotando menor rentabilidad. La posible explicación es la mayor productividad por hectárea, los precios de mercado para productos de cultivos de ciclo largo (frutales) y los menores costos de producción de Tocavita. 
Tabla X. Desempeño de indicadores en los tres agroecosistemas.

\begin{tabular}{|c|c|c|c|}
\hline Indicador & Tocavita & Chaine & Juruvita \\
\hline $\begin{array}{l}\text { Área del } \\
\text { agroecosistema (ha) }\end{array}$ & 6,37 & 4,84 & 5,5 \\
\hline VPN $(\$)$ & 6.163 .600 & 1.431 .936 & 344.287 \\
\hline Relación B/C & 1,3 & 1,05 & 1,05 \\
\hline Cobertura vegetal $(\%)$ & 82 & 60 & 62 \\
\hline $\begin{array}{l}\text { Disponibilidad de agua } \\
\left(\mathrm{m}^{3} / \mathrm{h}\right)\end{array}$ & 170,9 & 207 & 0,53 \\
\hline $\begin{array}{l}\text { Presión plagas y } \\
\text { enfermedades (en } \\
\text { escala de } 1 \text { a } 5 \text { ) }\end{array}$ & 3,5 & 4,6 & 4,2 \\
\hline $\begin{array}{l}\text { Calidad del suelo (en } \\
\text { escala de } 1 \text { a } 5 \text { ) }\end{array}$ & 3,75 & 2,35 & 2,75 \\
\hline $\begin{array}{l}\text { Diversidad biológica } \\
\text { (índice de Shannon, } \\
\text { valor entre } 0 \text { y } 5 \text { ) }\end{array}$ & 2,55 & 1,79 & 1,0 \\
\hline $\begin{array}{l}\text { Uso de semillas locales } \\
\text { (en escala de } 1 \text { a } 5 \text { ) }\end{array}$ & 4 & 3 & 4 \\
\hline $\begin{array}{l}\text { Empleo generado } \\
\text { (hectárea/año) }\end{array}$ & 155,8 & 126 & 42,7 \\
\hline $\begin{array}{l}\text { Participación en } \\
\text { asociaciones (\%) }\end{array}$ & 50 & 66 & 33 \\
\hline $\begin{array}{l}\text { Capacidad innovación } \\
\text { tecnológica (en escala } \\
\text { de } 1 \text { a } 5 \text { ) }\end{array}$ & 3 & 2 & 1 \\
\hline $\begin{array}{l}\text { Capacitación (en } \\
\text { escala de } 1 \text { a 5) }\end{array}$ & 5 & 5 & 2 \\
\hline $\begin{array}{l}\text { Dependencia insumos } \\
\text { externos (\%) }\end{array}$ & 67,1 & 74,4 & 85,8 \\
\hline $\begin{array}{l}\text { Capacidad de ahorro } \\
\text { (en escala de } 1 \text { a } 5 \text { ) }\end{array}$ & 4 & 2 & 4 \\
\hline $\begin{array}{l}\text { Autosuficiencia } \\
\text { alimentaria (\%) }\end{array}$ & 50 & 38,3 & 21 \\
\hline
\end{tabular}




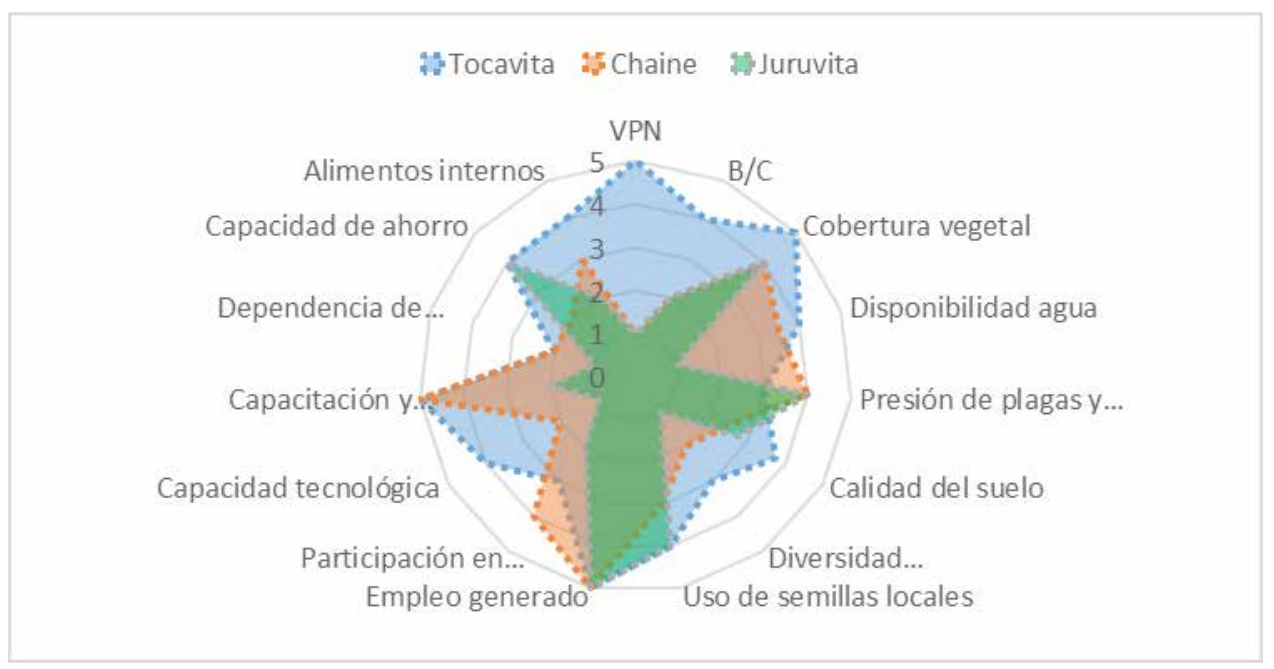

Figura 3. Mapa multicriterio desempeño de indicadores.

En la Figura 4 se presenta el análisis del nivel de sustentabilidad de los agroecosistemas a partir del comportamiento de los criterios de evaluación que se plantearon al inicio de esta investigación. Se muestra el promedio simple de los indicadores que forman parte de cada criterio. El criterio Retorno presenta las mayores diferencias entre los agroecosistemas, logrando en Tocavita un desempeño del $90 \%(4,5$ en la escala valorativa propuesta), mientras que en Chaine y Juruvita solamente logran un $30 \%$ (1,5 en la escala); esta diferencia se debe a la mayor rentabilidad y la mejor relación Beneficio/Costo. Similar comportamiento se presenta en el criterio Autosuficiencia, donde Tocavita logra un desempeño del $66 \%$ (3,3 en la escala), mientras que los otros agroecosistemas solo logran el $46 \%$ (2,3 en la escala). Lo anterior debido, entre otros aspectos, al aporte de alimentos del agroecosistema a la dieta del núcleo familiar. De la misma forma, el criterio Capacidad de cambio presenta evidentes diferencias entre los agroecosistemas, especialmente entre Tocavita y Juruvita; el primero alcanza un desempeño del $80 \%$, y el segundo, un $30 \%(4,0$ y 1,5 en la escala, respectivamente), elemento que presupone mayor disposición de Tocavita para la adopción y aplicación de tecnologías y prácticas que coadyuvan a aumentar los niveles de sustentabilidad (Figura 4).

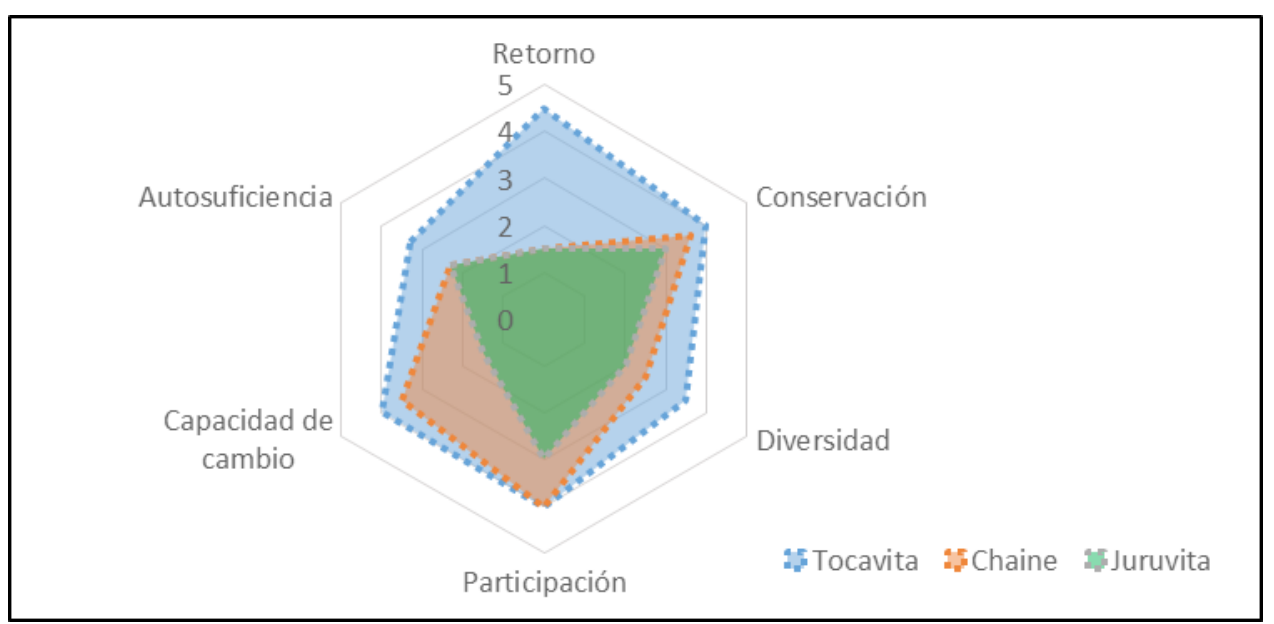

Figura 4. Mapa multicriterio Desempeño de criterios de evaluación. 
Los anteriores resultados son concordantes con otros trabajos de investigación que, mediante el método MESMIS u otros sistemas que evalúan la sustentabilidad (sostenibilidad) de agroecosistemas agrícolas tradicionales, han demostrado que los indicadores de fertilidad y biodiversidad de sistemas de producción tradicionales, tienen mejor comportamiento respecto de estos mismos criterios en sistemas de producción altamente intervenidos $(24,25)$.

Los resultados de la presente investigación sugieren que los sistemas de producción con altos niveles de biodiversidad, usos de semillas locales e incorporación de prácticas de protección de suelo y agua inciden positivamente en la permanencia del núcleo familiar alrededor de actividades agropecuarias, generando mayores niveles de rentabilidad que, a su vez, disminuyen la probabilidad de migración de los integrantes de la familia rural, contribuyendo así a preservar la cultura e idiosincrasia campesina y fortalecer la seguridad y la soberanía alimentaria de la región y del país.

\section{Conclusiones}

Los tres agroecosistemas campesinos evaluados (Tocavita, Chaine y Juruvita) en la microcuenca del río Cormechoque, usando el Marco para la Evaluación de Sistemas de Manejo de Recursos Incorporando Indicadores de Sustentabilidad (MESMIS), tienen características similares en el componente biofísico, pero presentan diferencias en aspectos tecnológicos y de manejo de las actividades agropecuarias, con diferencias tanto en la agrobiodiversidad y la productividad como en la rentabilidad.

El agroecosistema Tocavita supera a los de Chaine y Juruvita en todos los criterios evaluados; en el criterio Retorno obtiene un valor de 4,5 en la escala propuesta (de 1 a 5), mientras que los otros están cerca de 1,0.

Los desempeños más bajos los presenta el agroecosistema Juruvita, que alcanza, además de los ya mencionados, un valor de 2,0 en los criterios
Conservación y Participación en asociaciones locales. Este desempeño, posiblemente, es el resultado del bajo nivel de oferta ambiental expresado en los indicadores disponibilidad de agua, capacidad de recarga, calidad del suelo y biodiversidad, así como de las deficientes prácticas tecnológicas valoradas a través de los indicadores cobertura del suelo, incidencia de plagas y enfermedades y uso de semillas locales. Tal comportamiento, posiblemente, incide en el bajo desempeño de los indicadores generación de empleo, uso de mano de obra familiar y capacidad de ahorro, el cual impide un adecuado comportamiento en el indicador capacidad tecnológica, que a su vez se refleja en la alta dependencia de insumos externos.

El agroecosistema Tocavita tiene el mejor nivel de sustentabilidad en razón al desempeño del grupo de indicadores que dan cuenta de la gestión de la oferta ambiental (disponibilidad de agua, biodiversidad y calidad de suelo, entre otros), así como de prácticas culturales (cobertura vegetal, uso de semillas locales, capacitación y dependencia de insumos externos) que impactan positivamente el grupo de indicadores de rentabilidad del ejercicio agropecuario (VPN, relación $\mathrm{B} / \mathrm{C}$ ), estimulando la mayor permanencia del núcleo familiar en actividades agropecuarias dentro del agroecosistema.

Los resultados sugieren la interdependencia entre prácticas agrícolas en los agroecosistemas con sus condiciones biofísicas y la situación socioeconómica de la familia rural. Juruvita, por ejemplo, realiza prácticas con bajo nivel de desempeño (en la escala de 1 a 5), que han deteriorado su oferta ambiental y han menguado su capacidad productiva y la posibilidad de tener una mejor condición socioeconómica para sus integrantes, que los impulsa a abandonar las actividades agropecuarias $y$, posiblemente, a migrar a centros urbanos. Por lo tanto, la sustentabilidad de los agroecosistema está dada por la condición cultural de la familia rural, procurando la mayor o menor generación de beneficios ambientales y económicos que determinan la persistencia en el tiempo de este sistema de agricultura familiar. 


\section{Referencias}

(1) León Sicard TE. Medio ambiente, tecnología y modelos de agricultura en Colombia - Hombre y Arcilla. Bogotá: ECOE - Universidad Nacional de Colombia - Instituto de Estudios Ambientales; 2007.

(2) Gobernación de Boyacá. Superación de la pobreza extrema rural en Boyacá: Estrategia tierra viva. Oficina de planeación. (en línea) 2011. Disponible en: http://ennova.co/paginas/ observatorio/wp-content/uploads/2014/05/ pub-superacion-de-la-pobreza-extrema-rural. pdf.

(3) Altieri M. Construyendo resiliencia socio-ecológica en agroecosistemas: algunas consideraciones conceptuales y metodológicas. En: Nicholls C., Ríos L., Altieri M. (eds.): Agroecología y resiliencia ecológica: adaptándose al cambio climático, pp. 94-104. Medellín: Legis; 2013.

(4) Altieri M., Toledo VM. La Revolución Agroecológica de América Latina: rescatar la naturaleza, asegurar la soberanía alimentaria y empoderar al campesino. El otro derecho, 2010; 42(1): 163- 201.

(5) Pengue W. La Economía Ecológica y el desarrollo en América Latina. En: León y Altieri. Vertientes del pensamiento agroecológico: Fundamentos y aplicaciones, p. 125. Bogotá: Universidad Nacional de Colombia. Instituto de Estudios Ambientales - IDEA; 2010.

(6) Marco para la Evaluación de Sistemas de Manejo de recursos naturales incorporando Indicadores de Sustentabilidad. (en línea) 2012; Fecha de acceso 10 abril de 2015; Disponible en: http://mesmis.gira.org.mx/es/static/mesmis_framework.

(7) Herrera F., Rucks S., Moro B. Informe sobre el estado de avance de los objetivos de desarrollo del Milenio - Boyacá. (en línea) 2012. Disponible en http://www.pnud.org.co/2012/ odm2012/odm_boyaca.pdf.
(8) Escobar G., Berdagué L. Tipificación de sistemas de producción agrícola. Red internacional de metodología de investigación de sistemas de producción (RIMISP). Santiago de Chile.1990.

(9) Astier M., García L., Galván-Miyoshi Y., González-Esquivel CE, Masera O. R. Assessing the Sustainability of Small Farmer Natural Resource Management Systems. A Critical Analysis of the MESMIS Program. Ecology and Society. 2012; 17(3): 25. DOI: http://dx.doi. org/10.5751/ES-04910-170325.

(10) Alves EP, Silva LD, Oliveira Neto ND, Barrella TP, Santos HS. Economic analisys of a coffee-banana system of a family-based agriculture at the atlantic forest zone, Brazil. Ciência E Agrotecnologia. 2015; 39(3): 232239. DOI: https://dx.doi.org/10.1590/S141370542015000300004.

(11) Puentes GA. Formulación y evaluación de proyectos agropecuarios. Bogotá: Ecoe; 2011.

(12) Sandoval A., Ospina CE. Sustentabilidad ambiental en el manejo del agua y del suelo en la producción de berries. Los casos de México y Colombia. 2011; Ambiente y Desarrollo, 15(28), 99-122.

(13) Souza RF. Effect of management systems and cover crops on organic matter dynamics of soil under vegetables. Revista Brasileira de Ciência do Solo. 2014; 38(3): 923-933. DOI: http://dx.doi.org/10.1590/ S0100-06832014000300024.

(14) Martínez GF, Sosa PF. Comportamiento de la humedad del suelo con diferente cobertura vegetal en la cuenca La Esperanza. Tecnología y Ciencia del Agua. 2010; 1(3): 89-103.

(15) Instituto Colombiano Agropecuario. Manejo fitosanitario del cultivo de la papa (pdf en línea) 2011. Disponible en: http://www.ica.gov. co/getattachment/b2645c33-d4b4-4d9d-84ac197c55e7d3d0/Manejo-fitosanitario-del-cultiva-de-la-papa-nbsp;-.aspx. 
(16) International Potato Center. Manejo de gusano blanco. (en línea) 2005. Disponible en: http://cipotato.org/region-quito/informacion/ inventario-de-tecnologias/manejo-de-gusano-blanco-de-la-papa.

(17) Lafuente IC. Control biológico de mosca minadora. INIAP Archivo Historico. (en línea) 2002. Disponible en: http://books.google. com/books?id = hJgzAQAAMAAJ\&pgis $=1$.

(18) Orozco DJ, Flores CM, Sanabria YR. Indicadores químicos de calidad de suelos en sistemas productivos del Piedemonte de los Llanos Orientales de Colombia. Acta Agronómica, 2015; 64(4): 302-307.

(19) Pla Laura. Biodiversidad: Inferencia basada en el índice de Shannon y la riqueza. INCI 2006; 31(8): 583-590.

(20) Golicher DJ, O'Hara RB, Ruiz-Montoya L., Cayuela L. Lifting a veil on diversity: A Bayesian approach to fitting relative-abundance models. Ecological Applications, 2006; 16(1): 202-212. DOI: http://dx.doi.org/10.1890/041599.
(21) Golicher DJ. ¿Cómo cuantificar la diversidad de especies? Documento de trabajo. (en línea) 2012; p. 18. Disponible en: http://www. dfpd.edu.uy/cerp/cerp_norte/cn/Biologia/BIODIV/Como\% 20cuantificar\% 20la \% 20diversidad, \%20algunos \%20ejercicios.pdf.

(22) Ku V., Pool L., Mendoza J., Aguirre E. Propuesta metodológica para evaluar proyectos productivos con criterios locales de sustentabilidad en Calakmul, México. Avances en Investigación Agropecuaria. 2013; 17(1): 9-34.

(23) Aguilar Jiménez C., Tolón Becerra A., Lastra Bravo X. Evaluación integrada de la sostenibilidad ambiental, económica y social del cultivo de maíz en Chiapas, México. (Spanish). Rev. Fac. Cienc. Agrar., Univ. Nac. Cuyo. 2011; 43(1): 155-174.

(24) Pérez LB, Esquivel CG, Hernández LG. Evaluación de la sustentabilidad de dos agroecosistemas campesinos de producción de maíz y leche, utilizando indicadores. Livestock Research for Rural Development, 2005: 17(7).

(25) Aguirre S., Chiappe M. Evaluación de la sustentabilidad en predios hortícolas salteos. Agrociencia Uruguay, 2009; 13(1): 38-47. 